\title{
CANCER
}

\section{Subcutaneous or intrahepatic injection of suicide gene modified tumour cells induces a systemic antitumour response in a metastatic model of colon carcinoma in rats}

\author{
V Pierrefite-Carle, P Baqué, A Gavelli, N Brossette, D Benchimol, A Bourgeon, \\ M C Saint Paul, P Staccini, B Rossi
}

See end of article for authors' affiliations

....................

Correspondence to: $\checkmark$ Pierrefite-Carle, Unité INSERM 364, Faculté de Médecine, Avenue de Valombrose, 06107 Nice cédex 2, France; pierrefi@unice.fr

Accepted for publication 5 June 2001

\begin{abstract}
Background: Suicide gene therapy consists of the transfer into tumour cells of a "suicide" gene that can convert a non-toxic compound into a lethal drug. Expression of the cytosine deaminase gene leads to the conversion of the non-toxic compound 5-fluorocytosine to 5-fluorouracil. We have recently shown that "suicide cell based vaccination" consisting of intrahepatic injection of cytosine deaminase expressing colon cancer cells followed by 5 -fluorocytosine treatment induces regression of a distant wild-type liver tumour in rats.

Aims: This study was conducted to test if (i) a distant bystander effect on a liver tumour can be induced after subcutaneous suicide cell based vaccination and (ii) suicide cell based vaccination is efficient in limiting tumour dissemination to extrahepatic compartments.

Methods: An aggressive variant of rat colon carcinoma cells was selected after successive passages in vitro. Rats carrying an experimental liver "metastasis" generated by injection of these cells were vaccinated by subcapsular or subcutaneous injection of cytosine deaminase expressing cells followed by 5-fluorocytosine treatment.

Results: Subcutaneous and subcapsular vaccination induced $70 \%$ regression in the median volume of the pre-established liver tumour $(p=0.001)$ and abolished tumour dissemination compared with control animals.

Conclusions: This study has compared for the first time the efficiency of subcutaneous and intrahepatic suicide cell based vaccination in a metastatic colorectal carcinoma model in rats. The results indicate that both modes of vaccination are equally efficient in inducing a systemic antitumour response, suggesting that this strategy is a powerful approach against the development and dissemination of metastatic colon carcinoma.
\end{abstract}

S uicide gene therapy is based on the introduction into tumour cells of a non-mammalian gene encoding an enzyme that converts a non-toxic prodrug into a lethal drug. The cytosine deaminase (CD) gene of Escherichia coli transforms the antifungal prodrug 5-fluorocytosine (5-FC) into the widely used chemotherapeutic agent 5-fluorouracil (5-FU). This suicide strategy has several advantages: (i) it allows intratumoral chemotherapy, avoiding all of the toxic side effects generated by systemic 5-FU administration; (ii) its compensates for the poor gene transfer efficiency by the existence of a "local bystander" effect, known to induce regression of tumour although only a fraction of tumour cells express the suicide gene ${ }^{12}$; and (iii) its allow regression of distant unmodified tumour via a "distant bystander effect", induced by immune system stimulation during 5-FU mediated regression of the suicide gene modified tumour. ${ }^{3-11}$ This latter effect is of crucial importance for the treatment of disseminated metastasis as introduction of the suicide gene into a single tumour can theoretically result in regression of all metastases.

We have shown that introduction of the $\mathrm{CD}$ gene into a rat colon carcinoma cell line DHD/K12/PROb (or PROb) conferred 5-FC sensitivity in vitro. ${ }^{10}$ Intrahepatic injection of these CD expressing tumour cells (PRObCD) in syngenic rats resulted in the formation of an experimental liver suicide "metastasis" which dramatically regressed on 5-FC treatment. Regression of the 5-FC sensitive tumour conferred resistance to a new wild-type PROb tumour cell challenge in treated animals. ${ }^{9}{ }^{10}$ Moreover, a five day old microscopic PROb liver metastasis can be eradicated ( $45 \%$ of rats) or markedly decreased (55\% of rats) after intrahepatic injection of CD expressing tumour cells and 5-FC treatment.9 These results indicate the existence of a potent distant bystander effect generated after suicide cell based vaccination in a distant site of the same organ. Given the fact that 5-FC has no effect on PROb tumours, ${ }^{1012}$ we analysed the immune reaction triggered by vaccination and showed that the antitumour immune response was mediated by natural killer cells. ${ }^{9}$ A recent study of Lechanteur et al using the same PROb colon carcinoma animal model, demonstrated that injection of irradiated PROb tumour cells failed to induce any antitumoral immunity. ${ }^{12}$ These data justify the use of CD expressing tumour cells as a vaccine and suggest that in situ destruction of PRObCD cells is required for the establishment of an appropriate antitumour immune response.

The aim of our study was to determine the efficiency of suicide cell based vaccination in an advanced metastatic colon cancer model and to compare intrahepatic and subcutaneous modes of vaccination in this context.

Abbreviations: $\mathrm{CD}$, cytosine deaminase; 5-FC, 5-fluorocytosine; 5-FU, 5-fluorouracil; tk, thymidine kinase. 

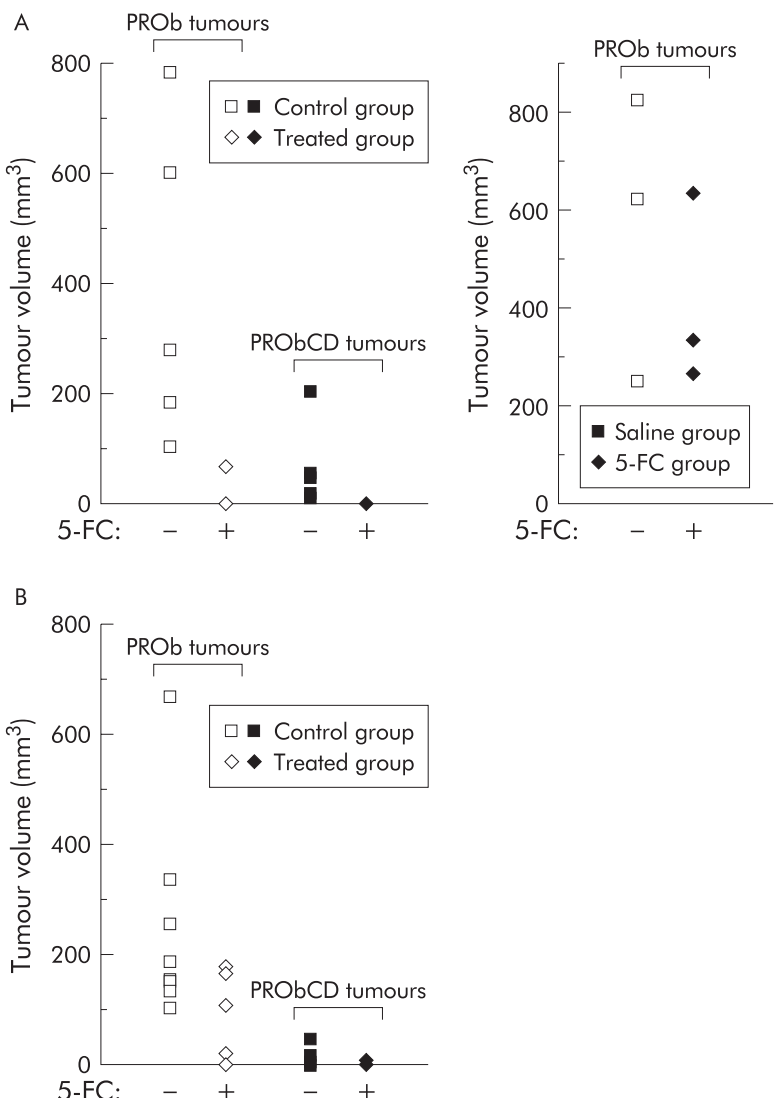

Figure 1 (A) Intrahepatic suicide cell based vaccination in an advanced liver metastasis model. Left: five days after subcapsular PROb tumour cell injection in the right liver lobe, all rats received subcapsular injection of PRObCD tumour cells in the left liver lobe. Animals were then either treated $(+; n=7)$ or not treated $(-; n=7)$ with 5-fluorouracil $(5-\mathrm{FC})$ for 30 days. The volumes of the PROb and PRObCD liver tumours in each group at the end of treatment are presented for the control and treated groups. Right: five days after unique subcapsular PROb tumour cell injection in the right liver lobe, rats were treated with saline or 5-FC for 30 days. The volumes of the PROb liver tumours in both groups at the end of the treatment are presented for the saline treated and 5-FC treated groups. (B) Subcutaneous suicide cell based vaccination in an advanced liver metastasis model. Five days after subcapsular PROb tumour cell injection in the right liver lobe, all rats received subcutaneous injection of PRObCD tumour cells. Animals were then either treated $(n=9)$ or not treated $(n=10)$ with 5-FC for 30 days. The volumes of the hepatic PROb and subcutaneous PRObCD tumours in each group at the end of the treatment are presented for the control and treated groups.

\section{MATERIAL AND METHODS}

\section{Cell culture}

DHD/K12/PROb (PROb) cells are a colon carcinoma cell line originating from a chemically induced colon cancer in BDIX rats. ${ }^{13}$ These cells are poorly immunogenic and induce progressive and metastatic tumours in syngenic hosts. ${ }^{14}$ The cells were maintained in Dulbecco's modified Eagle's medium (Biowhittaker) supplemented with 10\% fetal calf serum. PRObCD cells, generated by transfection of pCD $\beta$ geo plasmid expressing the $\mathrm{CD}$ gene $^{910}$ in the $\mathrm{PROb}$ cell line, were maintained in the same medium in the presence of G418 at $100 \mathrm{\mu g} / \mathrm{ml}$. A more aggressive variant of PROb cells was obtained after 40 passages of the parental cell line in vitro.

\section{Vaccination protocol}

For all experiments, we used adult BDIX male rats weighting 180-250 g (Iffa Credo, L'arbresle, France). All surgical procedures and animal care were in accordance with
A

Vaccination

(PRObCD cells injection)

Sacrifice

D5

D35

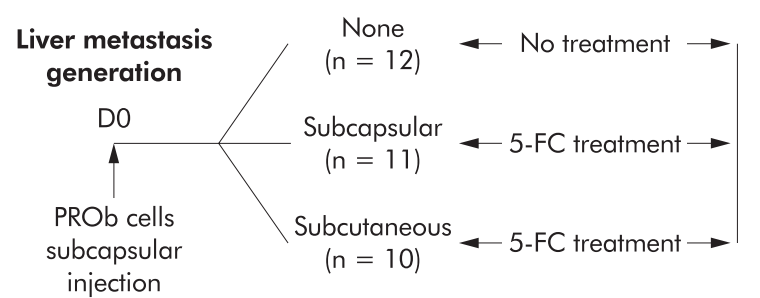

B

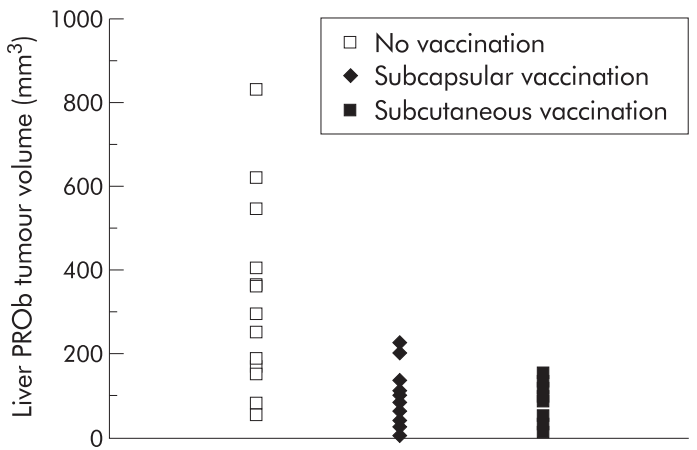

Figure 2 (A) Suicide cell based vaccination protocol. Five days after intrahepatic wild-type tumour cell injection, rats were randomised to one of three groups and treated as indicated. (B) Comparison of the therapeutic effect of subcapsular or subcutaneous vaccination on a pre-existing wild-type liver tumour. Volumes of PROb liver tumours in each group at the end of the treatment are presented for the control group, subcapsular vaccination group, and subcutaneous vaccination group.

institutional guidelines. All animals were randomly assigned to treatment.

Experimental liver metastases were generated by subcapsular injection of $1.5 \times 10^{6}$ PROb cells or variant PROb cells in the right lobe. Injection of variant tumour cells under the liver capsule of syngenic rats induced within 30 days a threefold larger (approximately $\mathrm{l} \mathrm{cm}$ diameter) hepatic tumour compared with injection of parental PROb cells. Five days after tumour cell injection, animals had surgery and the presence of the tumour was examined. In the case of intrahepatic vaccination, animals received an injection of $1.5 \times 10^{6} \mathrm{PRObCD}$ cells under the liver capsule in the left lobe (subcapsular vaccination) and were randomised to one of two groups. In the control group, rats were left untreated. In the treated group, 30 day treatment with 5-FC was initiated 24 hours after PRObCD cell injection.

In the case of subcutaneous vaccination, the animals received an injection of $1.5 \times 10^{6}$ PRObCD cells in the flank and were randomised to one of two groups. In the control group, rats were left untreated. In the treated group, animals were treated with 5-FC for 30 days, 24 hours after PRObCD cell injection.

For both vaccination modes, rats were sacrificed at the end of 5-FC treatment. Tumour volumes were calculated using the formula $a b^{2} \pi / 6$, where a refers to the largest diameter and $b$ to the perpendicular to $a$.

\section{5-FC treatment}

5-FC (Produits Roche, France) was dissolved in saline (15 $\mathrm{mg} / \mathrm{ml}$ ) and rats received three daily intraperitoneal injections of 5-FC ( $800 \mathrm{mg} 5-\mathrm{FC} / \mathrm{kg}$ body weight) for 30 days.

\section{Statistical analyses}

Results are expressed as median (95\% confidence intervals (CI) and range) and comparison of tumour volumes between 
Table 1 Presence of wound tumour nodes and peritoneal carcinomatosis in the different vaccination groups of rats

\begin{tabular}{lllll}
\hline Vaccination & Wound nodes & & \multicolumn{2}{l}{ Peritoneal carcinomatosis } \\
\hline None & $12 / 12(100 \%)$ & $p=0.0001$ & $7 / 12(58 \%)$ & $p=0.03$ \\
Subcutaneous & $2 / 10(20 \%)$ & $p=0.005$ & $1 / 10(10 \%)$ & $p=0.004$ \\
Subcapsular & $5 / 11(45 \%)$ & & $0 / 11(0 \%)$ & \\
\hline
\end{tabular}

$p$ values for comparison with the control group using Fisher's exact test are given. Due to Bonferroni's correction, $p$ values were considered statistically significant when less than 0.017 .

vaccinated and control rats was performed using the Mann-Whitney test, which is a non-parametric two-tailed probability test. Qualitative analysis (presence of a tumour) was performed using the two tailed Fisher's exact test. All statistics were computed with MINITAB Inc. V12.2 (State College, Pennsylvania, USA). Due to Bonferroni's correction, $p$ values were considered statistically significant when less than 0.017 .

\section{RESULTS}

\section{Regression of macroscopic pre-established liver} metastases after suicide cell based vaccination

We have previously demonstrated the efficiency of suicide cell based vaccination in a microscopic liver metastasis model in rats. ${ }^{11}$ To determine the efficiency of this strategy in an advanced cancer situation, intrahepatic suicide cell based vaccination was performed in animals carrying a five day old experimental liver metastasis generated by subcapsular injection of a more aggressive variant of the PROb colon carcinoma cell line. After intrahepatic PRObCD cell injection, animals $(\mathrm{n}=14)$ were either not treated (control group, $\mathrm{n}=7$ ) or treated with 5 -FC for 30 days (treated group, $n=7$ ). The volumes of liver tumours in each group are presented in fig $1 \mathrm{~A}$ (left panel). In the case of PRObCD tumours, median volume was $53.3 \mathrm{~mm}^{3}$ (95\% CI 23-133; range 14.6-207) in the control group compared with $0 \mathrm{~mm}^{3}$ ( $95 \%$ CI $0-0.5$; range $0-1$ ) in the treated group $(\mathrm{p}=0.002)$. In the case of PROb tumours, median volume was $282.4 \mathrm{~mm}^{3}$ (95\% CI 146-784; range 105784 ) in the control group compared with $0.1 \mathrm{~mm}^{3}$ (95\% CI $0-33.5$; range $0-66.9)$ in the treated group $(\mathrm{p}=0.002)$. To confirm that PROb tumour regression was totally dependent on the concomitant destruction of PRObCD cells, liver PROb tumours were generated and the animals were treated with either saline or 5-FC for 30 days. As shown in fig lA (right panel), no difference was observed in tumour volume between the two groups, confirming that 5-FC by itself had no effect on the growth of PROb tumours. Taken together, these results indicate that intrahepatic suicide cell based vaccination was efficient in an advanced liver metastasis model. We then analysed if subcutaneous suicide cell based vaccination can be efficient in this macroscopic liver metastasis model. After subcutaneous PRObCD cell injection, animals $(n=19)$ were either not treated (control group, $\mathrm{n}=10$ ) or treated with 5-FC for 30 days (treated group, $n=9$ ). The volumes of the tumours in each group are presented fig 1B. In the case of PRObCD tumours, median volume was $0 \mathrm{~mm}^{3}$ (95\% CI 0-23.5; range $0-47$ ) in the control group and $0 \mathrm{~mm}^{3}$ (95\% CI 0-4.7; range 0-9.4) in the treated group, suggesting that subcutaneous PRObCD tumours regressed in the presence or absence of 5-FC treatment. However, a significant difference in the volumes of PROb liver tumours was observed between control and treated groups. Indeed, the median volume of PROb liver tumours was $222 \mathrm{~mm}^{3}$ (95\% CI 151-402; range 105-669) in the control group and $6.3 \mathrm{~mm}^{3}$ (95\% CI 3-109; range 0-179) in the treated group $(\mathrm{p}=0.004)$.
Comparison of intrahepatic and subcutaneous suicide cell based vaccinations

To compare the efficiency of subcapsular and subcutaneous suicide cell based vaccination, an experimental liver metastasis from a colon carcinoma was generated by subcapsular injection of variant PROb tumour cells in syngenic rats. Five days later, the presence of the tumour was checked and animals were randomised to one of three groups, as shown in fig $2 \mathrm{~A}$. In the control group $(\mathrm{n}=12)$, rats were left untreated. In the subcapsular $(n=11)$ or subcutaneous $(n=10)$ vaccination groups, animals were subjected to, respectively, intrahepatic or subcutaneous injection of PRObCD cells followed by 30 day treatment with 5-FC. The volumes of the liver tumours in each group are presented in fig 2B. In the control group, animals presented with large tumours with a median tumour volume of $303.5 \mathrm{~mm}^{3}$ (95\% CI 165.9-454.4; range 63.5-836.8). Both types of vaccination induced significant regression of the liver tumour, with a median tumour volume of $91.5 \mathrm{~mm}^{3}(95 \%$ CI 37.9-125.9; range 8.4-230.6) $(\mathrm{p}=0.001)$ for subcapsular vaccination and $99.4 \mathrm{~mm}^{3}$ (95\% CI 42.7-122.2; range $1-163.3$ ) $(p=0.001)$ for subcutaneous vaccination. There was no statistically significant difference between these two modes of vaccination. Subcapsular or subcutaneous PRObCD tumours efficiently regressed in both groups, with a median tumour
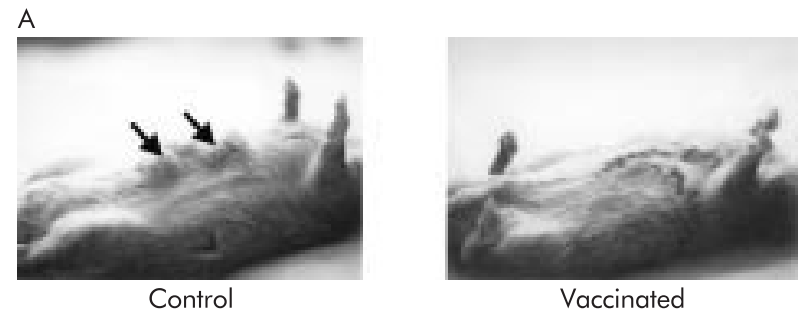

B

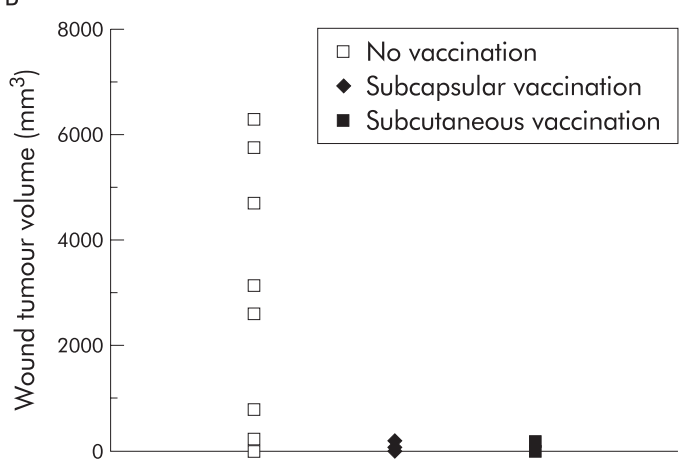

Figure 3 (A) Wound metastasis observed in control and vaccinated rats. The tumours are indicated by arrows. (B) Effect of suicide cell based vaccination on wound tumours. The volumes of the wound tumour nodules in each group are presented for the control group, subcapsular vaccination group, and subcutaneous vaccination group. 
volume of $0.5 \mathrm{~mm}^{3}$ (95\% CI 0.0-4.4; range 0-8.3) and $6 \mathrm{~mm}^{3}$ (95\% CI 2.3-26.0; range 0-38.4), respectively (data not shown).

\section{Subcutaneous or subcapsular suicide cell based vaccination inhibits tumour dissemination}

In addition to larger liver tumour generation, intrahepatic injection of aggressive variant PROb cells led to tumour dissemination to extrahepatic compartments. These metastases included peritoneal carcinomatosis and wound nodes originating from colonisation of the laparotomy cicatrix by tumour cells. Although all control animals exhibited wound tumour nodes, subcutaneous and subcapsular vaccination caused a significantly decreased occurrence of these metastases (table 1; fig 3A). There was no statistically significant difference between these two modes of vaccination. In addition, tumours exhibited by the vaccinated rats were significantly smaller than those of control animals (fig 3B). In the subcutaneous vaccination group, wound tumours had a median volume of $0 \mathrm{~mm}^{3}$ (95\% CI 0.0-92.3; range 0.0-184.6) compared with $779.3 \mathrm{~mm}^{3}$ in the control group $(95 \%$ CI 165.0-3222; range 41.8-5753.0) $(\mathrm{p}=0.0007)$. Similarly, subcapsular vaccination resulted in a decreased tumour volume with a median of $0 \mathrm{~mm}^{3}$ (95\% CI 0.0-64.9; range 0.0-207.6) $(\mathrm{p}=0.0004)$. There was no statistically significant difference in wound tumour volumes after subcutaneous or subcapsular vaccinations. Both treatments also influenced tumour dissemination to the peritoneal compartment (table 1).

\section{DISCUSSION}

The high lethality associated with colon carcinoma is generally due to the appearance of metastases preferentially located in the liver but also in the lungs and peritoneum. In this regard, a clinically efficient gene therapy strategy should allow regression of the gene modified tumour, followed by triggering of a systemic antitumour immune response. A distant bystander effect, which leads to regression of distant unmodified tumours, is one of the more interesting aspects of suicide gene therapy for the treatment of metastatic cancers. This effect is induced by immune system stimulation in response to prodrug mediated destruction of the suicide tumour cells. We have previously demonstrated that regression of a microscopic pre-engrafted liver metastasis was observed after CD expressing tumour cell injection on the contralateral liver lobe and 5-FC treatment. ' Duration of the therapeutic effect was confirmed in survival experiments in which $40 \%$ of the vaccinated animals were definitively cured. ${ }^{91}$ However, although the dead vaccinated rats $(60 \%)$ presented a limited liver metastasis, they all exhibited extensive peritoneal tumour dissemination, suggesting that the distant bystander effect could be a local organ specific phenomenon. To address this question, we generated a more aggressive variant of the PROb colon carcinoma cell line, which induced after subcapsular injection both liver metastasis and tumour dissemination to extrahepatic compartments within 30 days. In the first experiment, we analysed the efficiency of intrahepatic suicide cell based vaccination in this advanced liver metastasis model. Regression of liver PRObCD tumours was dependent on 5-FC treatment, and a distant bystander effect, acting on hepatic PROb tumours, was observed in the treated group. These results confirmed the existence of a distant bystander effect even in a more aggressive tumour model. A similar distant bystander effect was also observed in rats receiving subcutaneous injection of PRObCD cells and 5-FC treatment. However, in this case, regression of subcutaneous PRObCD tumours was independent of 5-FC treatment, suggesting the triggering of different immune responses between skin and liver. 5-FC independent regression of $\mathrm{CD}$ expressing subcutaneous tumours was previously described by Mullen et al and Nanni et al in pulmonary metastases murine models..$^{15}{ }^{16}$ This observation suggests that expression of the CD gene itself contributes to generation of an immune reaction leading to destruction of the PRObCD tumour in skin but not liver. This effect could result from the recognition of the $\mathrm{CD}$ protein as a bacterial antigen. None the less, it is of particular interest that although PRObCD tumours regressed both in control and treated groups, regression of the PROb liver tumour was strictly dependent on 5-FC mediated regression of the PRObCD tumour. Given that 5-FC has no effect on PROb tumours, these data suggest that the death mechanism involved in the destruction of PRObCD tumour by 5 -FC plays a crucial role in sparking the antitumour immune response.

We then compared intrahepatic and subcutaneous vaccination modes in the same experiment. Subcapsular suicide cell based vaccination resulted in a $70 \%$ decrease in median tumour volume of the liver pre-established metastasis compared with untreated animals. In the control group, tumour dissemination resulted in the appearance of wound tumours and peritoneal carcinomatosis in $100 \%$ and $58 \%$ of animals, respectively. After intrahepatic vaccination, $54.5 \%$ of the vaccinated animals did not exhibit any wound tumours and $45.5 \%$ presented small tumours with a 780 -fold decrease in median tumour volume compared with control rats. Peritoneal carcinomatosis was inhibited in $100 \%$ of these animals. These results suggest that a distant bystander effect is not an organ specific event and that the immune reaction triggered by suicide gene therapy can impair tumour dissemination. This hypothesis was strengthened by the data obtained after subcutaneous vaccination. Indeed, subcutaneous vaccination induced a $67 \%$ decrease in median tumour volume of the liver tumour and abolished wound tumour formation in $80 \%$ of rats. Similarly, in the $20 \%$ vaccinated rats presenting a tumour, median tumour volume was markedly reduced ( 780 -fold). Even though the results were not statistically significant $(p>0.017)$, the occurence of peritoneal carcinomatosis was also reduced in these rats $(1 / 10(10 \%) v 7 / 12(58 \%)$ in control rats). These data suggest that peritoneal carcinomatosis, observed several months after vaccination in vaccinated rats that died in our previous study, most likely resulted from a relapse of the tumour rather than a lack of systemic immunity.

Two different studies reported that subcutaneous injection of tumour cells expressing CD and interleukin 6 genes ${ }^{15}$ or CD and interferon $\gamma$ genes $^{16}$ resulted in growth inhibition of a lung metastasis in mice. In the HSVl thymidine kinase (tk)/ ganciclovir suicide system, Santodonato et al demonstrated the existence of a systemic antitumour response after subcutaneous injection of tumour cells expressing interferon $\alpha$ and HSVtk in a mouse metastatic tumour model. ${ }^{17}$ Our study constitutes the first comparative analysis between two vaccination sites with regard to their efficiency in inducing an antitumour response. The results presented here indicate that a distant bystander effect is not an organ specific event and that a systemic immune reaction can be triggered by suicide gene therapy regardless of the mode of injection. The immune response resulting from destruction of CD expressing tumour cells can impair tumour dissemination to distant organs, such as the wound and the peritoneum. Taken together, these data suggest that intrahepatic or subcutaneous injection of CD expressing autologous tumour cells followed by 5 -FC administration could represent a powerful alternative therapeutic regimen for the treatment of patients with disseminated unresectable metastasis from colon carcinoma.

\section{ACKNOWLEDGEMENTS}

This work was supported by the Institut National de la Santé et de la Recherche Médicale, Ligue Départementale contre le Cancer and Association pour la Recherche sur le Cancer (ARC). The authors are indebted to Professor F Martin (Unité INSERM 517, Dijon, France) for kindly providing the $\mathrm{DHD} / \mathrm{K} 12 / \mathrm{PROb}$ (PROb) cell line. We thank Produits Roche (France) for supplying 5-FC. The expert technical assistance of R Grattery is gratefully acknowledged. 


\section{Authors' affiliations}

V Pierrefite-Carle, P Baqué, A Gavelli, N Brossette, B Rossi, Unité INSERM 364, IFR 50, Faculté de Médecine, Avenue de Valombrose,

06107 Nice cedex 2, France

D Benchimol, A Bourgeon, Service de Chirurgie Oncologique, Hôpita I'Archet II, 151 Avenue Saint Antoine de Ginestière, 06202 Nice cédex 3, France

M C Saint Paul, Service d'Anatomopathologie, Hôpital Pasteur, Avenue de Valombrose, 06107 Nice cedex 2, France

P Staccini, Biostatistiques et Informatique Médicale, Faculté de

Médecine, Avenue de Valombrose, 06107 Nice cédex 2, France

$\checkmark$ Pierrefite-Carle and $\mathrm{P}$ Baqué contributed equally to this work.

\section{REFERENCES}

1 Freeman SM, Abboud CN, Whartenby KA, et al. The "bystander effect": tumor regression when a fraction of the tumor mass is genetically modified. Cancer Res 1993:53:5274-83.

2 Huber BE, Austin EA, Richards CA, et al. Metabolism of 5-fluorocytosine to 5 -fluorouracil in human colorectal tumor cells transduced with the cytosine deaminase gene: significant antitumor effects when only a small percentage of tumor cells express cytosine deaminase. Proc Natl Acad Sci USA 1994;91:8302-6.

3 Dilber MS, Abedi MR, Bjorkstrand B, et al. Suicide gene therapy for plasma cell tumors. Blood 1996;88:2192-200.

4 Wilson KM, Stambrook PJ, Bi WL, et al. HSV-tk gene therapy in head and neck squamous cell carcinoma. Enhancement by the local and distant bystander effect. Arch Otolaryngol Head Neck Surg 1996; 122:746-9.

5 Bi W, Kim YG, Feliciano ES, et al. An HSVtk-mediated local and distant antitumor bystander effect in tumors of head and neck origin in athymic mice. Cancer Gene Ther 1997;4:246-52.

6 Kianmanesh AR, Perrin H, Panis Y, et al. "Distant" bystander effect of suicide gene therapy: regression of nontransduced tumors together with a distant transduced tumor. Hum Gene Ther 1997:8:1807-14.
7 Wei MX, Bougnoux P, Sacre-Salem B, et al. Suicide gene therapy of chemically induced mammary tumor in rat: efficacy and distant bystande effect. Cancer Res 1998;58:3529-32.

8 Hall SJ, Sanford MA, Atkinson G, et al. Induction of potent antitumor natural killer cell activity by herpes simplex virus-thymidine kinase and ganciclovir therapy in an orthotopic mouse model of prostate cancer. Cancer Res 1998;58:3221-5.

9 Pierrefite-Carle V, Baqué P, Gavelli A, et al. Cytosine deaminase/5-fluorocytosine-based vaccination against liver tumors: Evidence of distant bystander effect. J Natl Cancer Inst 1999;91:2014-9.

10 Pierrefite-Carle V, Baqué P, Gavelli A, et al. Regression of experimental liver tumor after distant intra-hepatic injection of cytosine deaminase-expressing tumor cells and 5-fluorocytosine treatment. Int J Mol Med 2000;5:275-8.

11 Pierrefite-Carle V, Gavelli A, Brossette N, et al. Re: Cytosine deaminase/5-fluorocytosine-based vaccination against liver tumors: Evidence of distant bystander effect. I Natl Cancer Inst 2000;92:494-5.

12 Lechanteur $C$, Moutschen M, Princen F, et al. Antitumoral vaccination with granulocyte-macrophage colony-stimulating factor or interleukin-12-expressing DHD/K12 colon adenocarcinoma cells. Cance Gene Ther 2000;7:676-82.

13 Martin F, Caignard A, Jeannin JF, et al. Selection by trypsin of two sublines of rat colon cancer cells forming progressive or regressive tumors. Int J Cancer 1983:32:623-7.

14 Caignard A, Genne P, Olsson NO, et al. F11C antigen: a membrane marker able to distinguish two regressive and progressive variants from a rat colon adenocarcinoma. Int J Cancer 1990;46:633-9.

15 Mullen CA, Petropoulos D, Lowe RM. Treatment of microscopic pulmonary metastases with recombinant autologous tumor vaccine expressing interleukin 6 and Escherichia coli cytosine deaminase suicide genes. Cancer Res 1996;56:1361-6.

16 Nanni P, De Giovanni C, Nicoletti G, et al. The immune response elicited by mammary adenocarcinoma cells transduced with interferon-gamma and cytosine deaminase genes cures lung metastases by parental cells. Hum Gene Ther 1998:9:217-24.

17 Santodonato L, D'Agostino G, Santini SM, et al. Local and systemic antitumor response after combined therapy of mouse metastatic tumors with tumor cells expressing IFN-alpha and HSVtk: perspectives for the generation of cancer vaccines. Gene Ther 1997;4:1246-55.

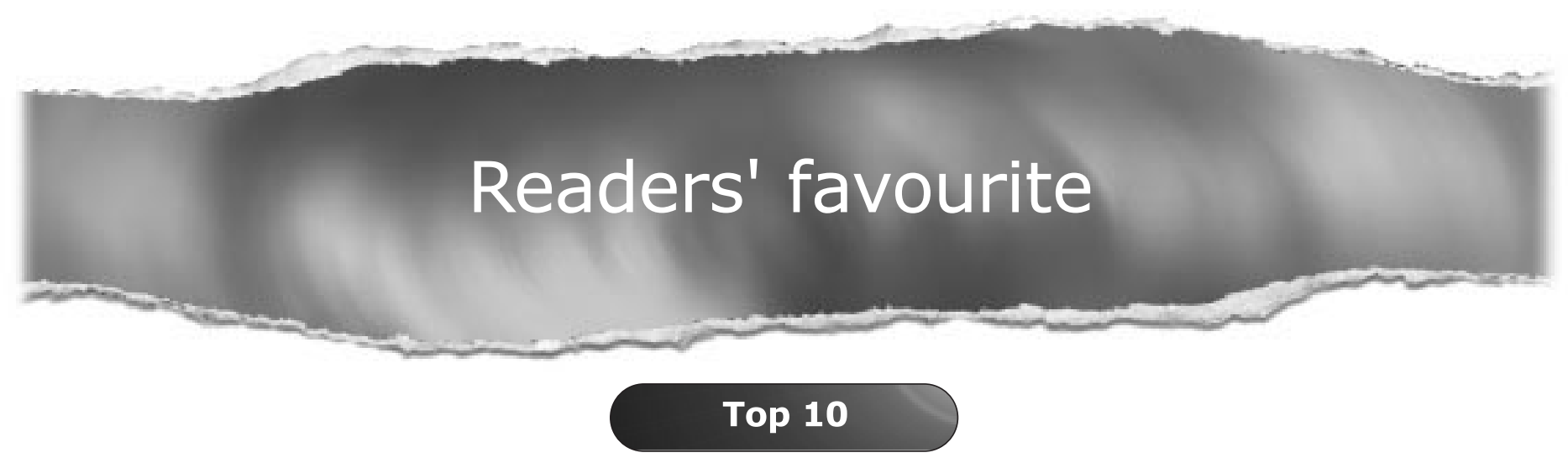

Click on the "Top 10" button on the homepage to see which are the best read articles each month

\section{www.gutjnl.com}

